Article

\title{
Development of an E-Health App for Lower Limb Postoperative Rehabilitation Based on Plantar Pressure Analysis
}

\author{
Xiao Cheng ${ }^{1}$, Xin $\mathrm{Mei}^{2}$, Yue Hu ${ }^{1}$, Yinfang Fang ${ }^{1}$, Shuai $\mathrm{Wu}^{1}$, Fengxiang You ${ }^{1,3, *}$ and \\ Shaolong Kuang ${ }^{1,3, *}$ \\ 1 Jiangsu Provincial Key Laboratory of Advanced Robotics, Soochow University, 215000 Suzhou, China; \\ 20164229028@stu.suda.edu.cn (X.C.); ENJJDHY@163.com (Y.H.); fangyinfang1020@163.com (Y.F.); \\ 20175229079@stu.suda.edu.cn (S.W.) \\ 2 The First Affiliated Hospital of Suzhou University, 215000 Suzhou, China; mainstar@126.com \\ 3 Collaborative Innovation Center of Suzhou Nano Science and Technology, Soochow University, \\ 215000 Suzhou, China \\ * Correspondence: Youfengxiang98@163.com (F.Y.); slkuang@suda.edu.cn (S.K.); Tel.: +86-137-7188-4496 (F.Y.); \\ +86-180-1311-5051 (S.K.)
}

Received: 30 March 2018; Accepted: 8 May 2018; Published: 11 May 2018

\begin{abstract}
The traditional postoperative rehabilitation training mode of lower limbs is mostly confined to hospitals or nursing sites. With the increase of postoperative patients, the current shortage of medical resources is obviously not satisfactory, and the medical costs are high, thus it is difficult to apply widely. A new mobile phone application (app) based on plantar pressure analysis is developed to fulfill the requirements of remote postoperative rehabilitation. It is designed, implemented, tested, and used for pilot experiment in conjunction with the system design methodology of the waterfall model. Preliminary testing and a pilot experiment showed that the app has realized basic functions and can achieve patient rehabilitation out of hospitals. The development of the app can shorten the hospitalization time of patients, reduce medical costs, and make up for the current shortage of medical resources. In the future, more experiments will be done to verify the effectiveness of the app.
\end{abstract}

Keywords: lower limb; mobile phone application; rehabilitation out of hospital; plantar pressure analysis

\section{Introduction}

Early rehabilitation training is important to patients who have undergone lower limb surgery, which can speed up the recovery of limb function, greatly reduce the rehabilitation process, and effectively prevent the occurrence of postoperative complications [1,2]. However, early postoperative rehabilitation training techniques are limited for the lack of sensor and evaluation methods. For example, all patients should be recumbent for a certain period of time after surgery to prevent from secondary injury in China. Another problem of this method is that it may bring about complications such as venous thrombosis and more serious consequences [3]. Thus, it has become a major challenge to select a suitable method for early sensor technique and evaluation of effectiveness after surgery to ensure better recovery of patients. Studies have shown that the health condition of a persons' lower limbs can be reflected by the persons' gait characteristics and plantar pressure distribution [4-6]. There are certain laws in the distribution of healthy persons' plantar pressure. Deformity and abnormal function will destroy the normal distribution of plantar pressure, showing different distribution laws [7]. Therefore, it is of great value to evaluate the rehabilitation effect of patients by combining rehabilitation training with plantar pressure. 
Moreover, most of the traditional patterns of lower limb postoperative rehabilitation training adopt physician-assisted manual training or gait rehabilitation robot assisted training, which is confined to hospital or nursing sites, and at least one physician or nurse is needed [8-10]. With the increase of postoperative patients, the current shortage of medical resources and professional rehabilitation physicians is obviously not satisfactory, and the medical costs are high [11-13]. Thus, more and more attention has been paid to the mode of treatment in the hospital and rehabilitation out of hospitals [14-16]. Currently, by using the plantar pressure with current telemedicine, some rehabilitation processes in the hospital can be transferred to communities or families by setting up a powerful information database. More importantly, it can improve medical efficiency and reduce the medical cost, but the information interaction between patients and doctors is a main challenge in this mode. With the development of "medical + internet" technology, researchers have done much work with tele-medicine methods [17,18]. In this study, an Android mobile phone application (app) is designed, through which information between patients and doctors can be transmitted immediately, such as downloading training plans to patients, uploading training data to doctors. Patients can also learn about their own training status, such as the training phase and training effect. In addition, patients can communicate with doctors via various ways such as voice, small video, and internet messaging, realizing the rehabilitation mode for the patient out of hospital and greatly reducing hospitalization time to compensate for inconvenience caused by the current shortage and imbalance of distribution of medical resources.

\section{Materials and Methods}

The waterfall model is the most frequently used model in software development. It divides the software development process into several stages. The latter stage receives the work object from the previous stage as input, and uses this input to implement the stage and give the work result of the stage as output to the next stage. At the same time, the testing result of system will be reviewed, if the result is not confirmed, it will return to the previous stages. The process is shown in Figure 1 [19]. Using the waterfall model, app development steps are as follows:

(1) App requirements analysis

(2) App design

(3) App implementation

(4) App testing

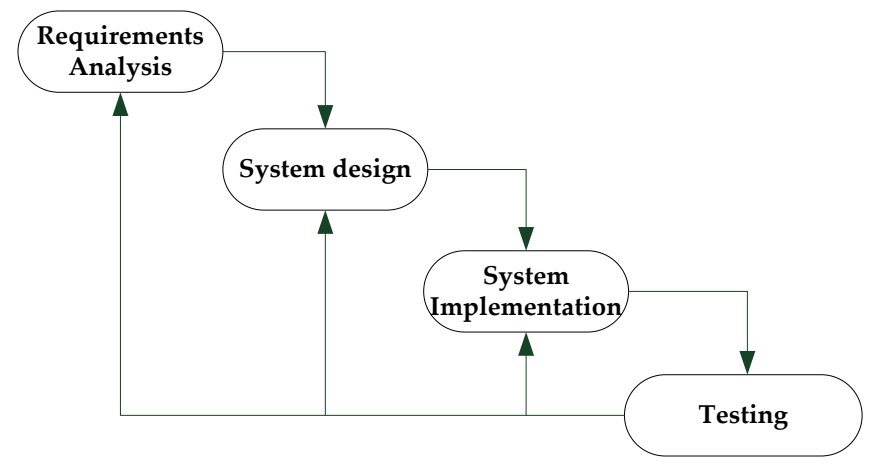

Figure 1. Waterfall model.

\subsection{App Requirements Analysis}

The app based on plantar pressure analysis enables doctors to know the training situation of patients out of hospital in real time and to give corresponding training instructions. By carefully combing and refining user needs, the main functions of the app are as follows: 
(1) Training plan management: Getting the training plan from the doctor and displaying it on the phone. The training plan can also be downloaded to the plantar pressure testing equipment via the app. The training plan includes the number of training days, daily times, maximum force, and minimum force in this phase and so on.

(2) Training data management: The real-time pressure during training and the record after this training can be obtained from the plantar pressure testing equipment and uploaded to the doctor's database. At the same time, the real-time pressure curve and the data analysis after training can be viewed in the app interface. The training record includes the maximum strength of this training, the number of times exceeding the maximum strength, average force, training time, etc.

(3) Instant communication function: Through this app, communication between doctors and patients can be performed in real time.

(4) Saving patients' basic information: If the patient has logged in, the patients can login automatically the next time when they enter the system. As an important indicator of the training plan, patients' detailed information need to be improved (age, weight, etc.) on the app and submitted to the doctor.

(5) Communication device selection: Through the app, the plantar pressure detecting device can be selected to communicate with the app.

\subsection{App Design}

The app design based on planar pressure analysis mainly includes the overall architecture design, database design, interaction design, module division, and functional design.

\subsubsection{Overall Architecture Design and Module Division}

An app is mainly divided into application, business, and data layers [20]. The overall architecture of the app is shown in Figure 2. The application layer is the interface of the app, including the login, training plan, and other pages. The business layer is the specific operation process of the app. The data layer stores user information, training plans, and other data.

\begin{tabular}{|c|c|c|c|c|c|c|c|c|}
\hline \multirow{3}{*}{$\begin{array}{c}\text { Overall } \\
\text { Archite } \\
\text { cture }\end{array}$} & \multirow{3}{*}{\begin{tabular}{|c|}
$\begin{array}{c}\text { Application } \\
\text { Layer }\end{array}$ \\
$\begin{array}{c}\text { Business } \\
\text { Layer }\end{array}$ \\
\end{tabular}} & $\log$ & $\begin{array}{l}\text { Training } \\
\text { Plan }\end{array}$ & \multicolumn{2}{|c|}{ Training Data } & \multicolumn{3}{|c|}{ Mine } \\
\hline & & \multicolumn{2}{|c|}{$\begin{array}{l}\text { Training Plan } \\
\text { Management }\end{array}$} & \multicolumn{2}{|c|}{ Training Data Management } & \multicolumn{3}{|c|}{ Personal Information Management } \\
\hline & & Acquire & Download & Get & Upload & $\begin{array}{c}\text { Instant } \\
\text { Messaging }\end{array}$ & $\begin{array}{l}\text { Wireless } \\
\text { Connection }\end{array}$ & $\begin{array}{l}\text { Integrate } \\
\text { Personal } \\
\text { Information }\end{array}$ \\
\hline & Data Layer & \multicolumn{7}{|c|}{ Basic Data } \\
\hline
\end{tabular}

Figure 2. The overall architecture.

According to the principle of 'high cohesion, low coupling', combined with the above-mentioned overall framework, the app can be divided into four major modules: registration and login module, training plan management module, training data management module, and 'mine' module. The functional block diagram is shown in Figure 3.

\subsubsection{Database Design}

Training for patients is not necessarily the real-time networking. If the training data were not saved in the training process, the doctor will not be able to get the patient's rehabilitation condition when checking training effect. The patient also wants to view the training plan, historical records, and other data in the absence of the network, so a mobile database is needed here to store data 
temporarily. The SQLite is used in this design because it is compact, convenient, and easy to develop. The data tables stored in the database include the user information table, training plan table, real-time data table, and historical record table. There are one-to-one or one-to-many relationships among the tables. During our study, one user corresponds to one piece of personal information, multiple training plans, multiple real-time data, and historical records.

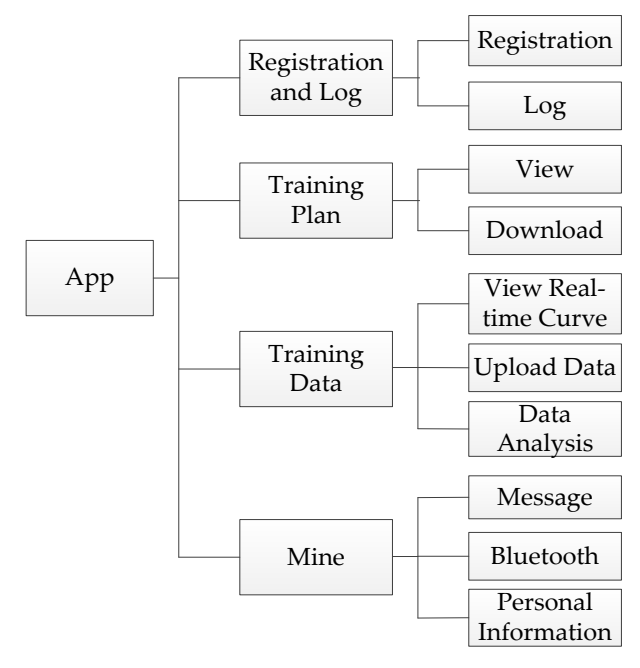

Figure 3. Functional block diagram.

\subsubsection{Interaction Design}

The interaction design is to build a medium of interaction between human users and the app. The user receives information from the machine through the senses of sight and hearing, and reacts after processing and making decisions. When the machine receives user commands, it will display after controlling and processing, so as to realize the human-machine information transmission. The human-machine system model is shown in Figure 4, which is a very important system between the human and machine.

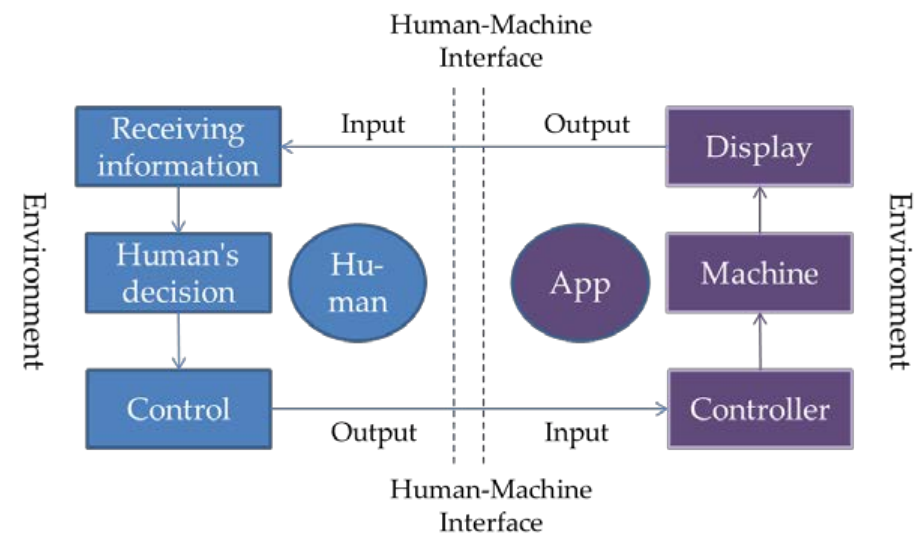

Figure 4. Human-machine interaction model.

The user-oriented method is used to develop the human-machine interface (HMI), making people work more effectively. The so-called user-oriented design is aimed at transferring message to people by machine and communicating effectively between them. Therefore, in order to make the machine more user-friendly, the APP is designed by using some UI design principles, such as consistency, clarity level of information showing, a simple and easy-to-understand interface layout, and contrast principle. The specific application is shown in Table 1. 
Table 1. The specific application of UI design principles.

\begin{tabular}{lll}
\hline \multicolumn{1}{c}{ Principle } & \multicolumn{1}{c}{ Explanation } & \multicolumn{1}{c}{ Application } \\
\hline $\begin{array}{l}\text { Clearly show the } \\
\text { level of information }\end{array}$ & $\begin{array}{l}\text { The user's attention span is very short, } \\
\text { it needs to guide the user to focus on the } \\
\text { core information in the shortest time to } \\
\text { complete the operation. }\end{array}$ & $\begin{array}{l}\text { A total of three layers are set for the app. The first } \\
\text { layer includes the training plan, training data, } \\
\text { and mine. The second layer includes the } \\
\text { directory of messages, Bluetooth, and personal } \\
\text { information. The third layer is mainly the details } \\
\text { of message, Bluetooth, and personal information. }\end{array}$ \\
\hline $\begin{array}{l}\text { Consistency } \\
\text { Consistent human-machine interface } \\
\text { allows users to transfer existing knowledge } \\
\text { and experience to new tasks to learn and } \\
\text { use the app faster. }\end{array}$ & $\begin{array}{l}\text { For the three components 'message', 'Bluetooth', } \\
\text { 'personal information' in 'mine', if you click the } \\
\text { interface layout } \\
\text { click any other part, there should be the same } \\
\text { event happen. }\end{array}$ \\
\hline $\begin{array}{l}\text { Contrast principle } \\
\text { The simplicity of the interface is to make the } \\
\text { the possibility of user error selection. }\end{array}$ & $\begin{array}{l}\text { The organization of the screen is from left to right, } \\
\text { from top to bottom, the controls of the interface } \\
\text { are designed using the space in the system to } \\
\text { make people understand at a glance. }\end{array}$ \\
\hline
\end{tabular}

\subsection{App Implementation and Testing}

\subsubsection{Development and Debugging}

According to the above design ideas, the app is developed with Android Studio as the main tool and Java as the programming language. The illustrative images of the app interface are shown in Figure 5. There are four modules:

(1) Registration and login module (as shown in Figure 5a): It is used for patients to register and login, which can protect the patient's information. After the patient registers, the app will send the patient information to the doctor database to use for patient information management and future login verification. At the same time, the app will save the data in the mobile database, and the next time the app is used, it will automatically log in.

(2) Training plan management module (as shown in Figure 5b): On the one hand, if the network is in good condition, the app can obtain the patient's training plan from the doctor's database and display it. At the same time, the latest data acquired can be stored on the mobile database. If not, the training plan stored in the mobile database can be read and displayed; on the other hand, the app can transmit the training plan to the plantar pressure testing equipment through wireless communication.

(3) Training data management module (as shown in Figure 5c): The module mainly uses wireless transmission to obtain and save the real-time pressure during the training process and the record after the training. At the same time, the training data is transmitted to doctor for the doctor viewing in the future. In addition, the module can perform a simple analysis of the training data and provide reasonable advice for the next training session.

(4) 'Mine' module (as shown in Figure 5d): The module consists of three parts: message, Bluetooth, and personal information. The 'message' part is to enable real time communication between the patient and the doctor, referring to instant messaging software such as QQ and MSN. The 'Bluetooth' part is a wireless communication method. This article uses Bluetooth as an example, and is mainly connected to the plantar pressure detection equipment through the socket protocol. The 'personal information' part is the information other than the patient's login information, such as name, gender, age, weight, etc. After submission, the data will be saved in the SQL database to the doctor's database. 


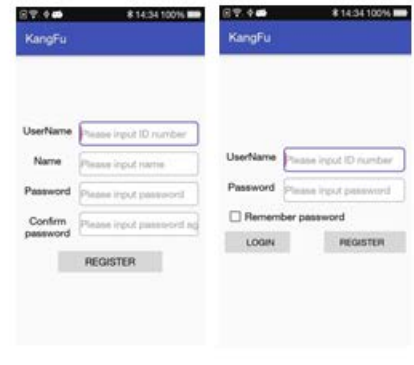

A

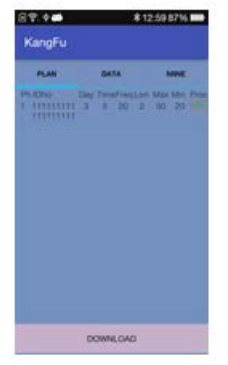

B

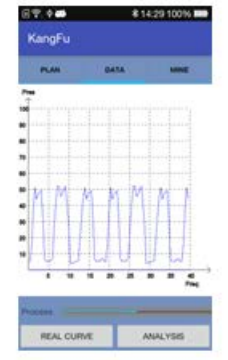

$\mathrm{C}$

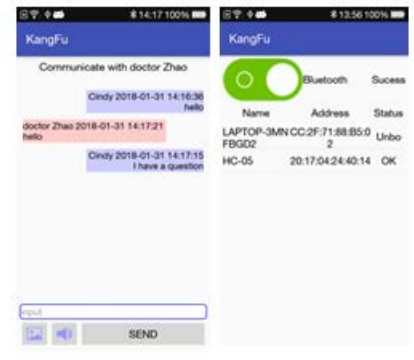

D

Figure 5. Illustrative images of the App interface. (A) Registration and Log; (B) Training plan; (C) Training data; (D) Mine.

\subsubsection{App Testing}

A mobile phone device with the type of OPPO R831S (OPPO Corporation, Dongguan, China) and Android 4.3 software is used to develop the app. The testing platform is set up with plantar pressure testing device and doctor interface, as shown in Figure 6. The processes are as follows:

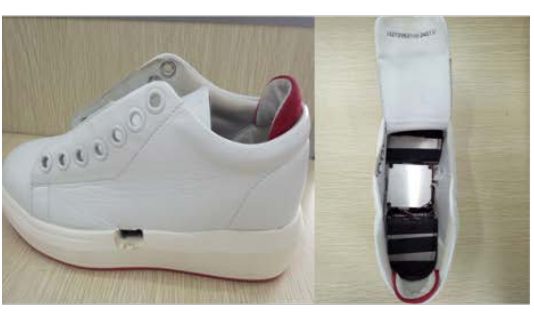

(A)

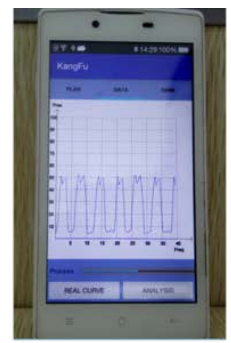

(B)

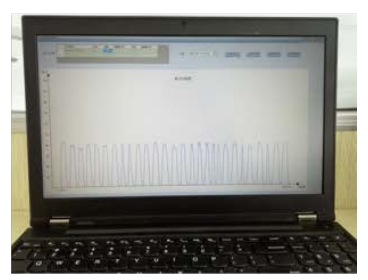

(C)

Figure 6. Testing system: (A) Plantar pressure testing device; (B) App; (C) Doctor interface.

(1) Firstly, registering and logging are on the start of the app (as shown in 'Registration and log in' Figure 7 step 1). After logging in, the training plan can be obtained from the doctor's database.

(2) In 'Mine', the plantar pressure testing device can be paired with App via 'Bluetooth' (as shown in 'Pairing' in Figure 7 step 2).

(3) The training plan can be downloaded to the plantar pressure testing device by click the 'Download' button in the 'Training plan' interface The patient will train according to the training plan (as shown in 'Download training plan' in Figure 7 step 3).

(4) During the training process, the real-time curve can be viewed in the 'training data' (as shown in 'Real-time curve' in Figure 7 step 4), and the progress of this training can also be seen in this figure.

(5) After each training session, the comparison between this training session, previous training sessions, and normal gait can be viewed, and a reasonable suggestion to the next training will be given (as shown in 'Data analysis' in Figure 7 step 5).

(6) In the event of a problem, the communication between patients and doctors can be performed in 'Message' interface in 'Mine' (as shown in the 'Message' in Figure 7 step 6). 


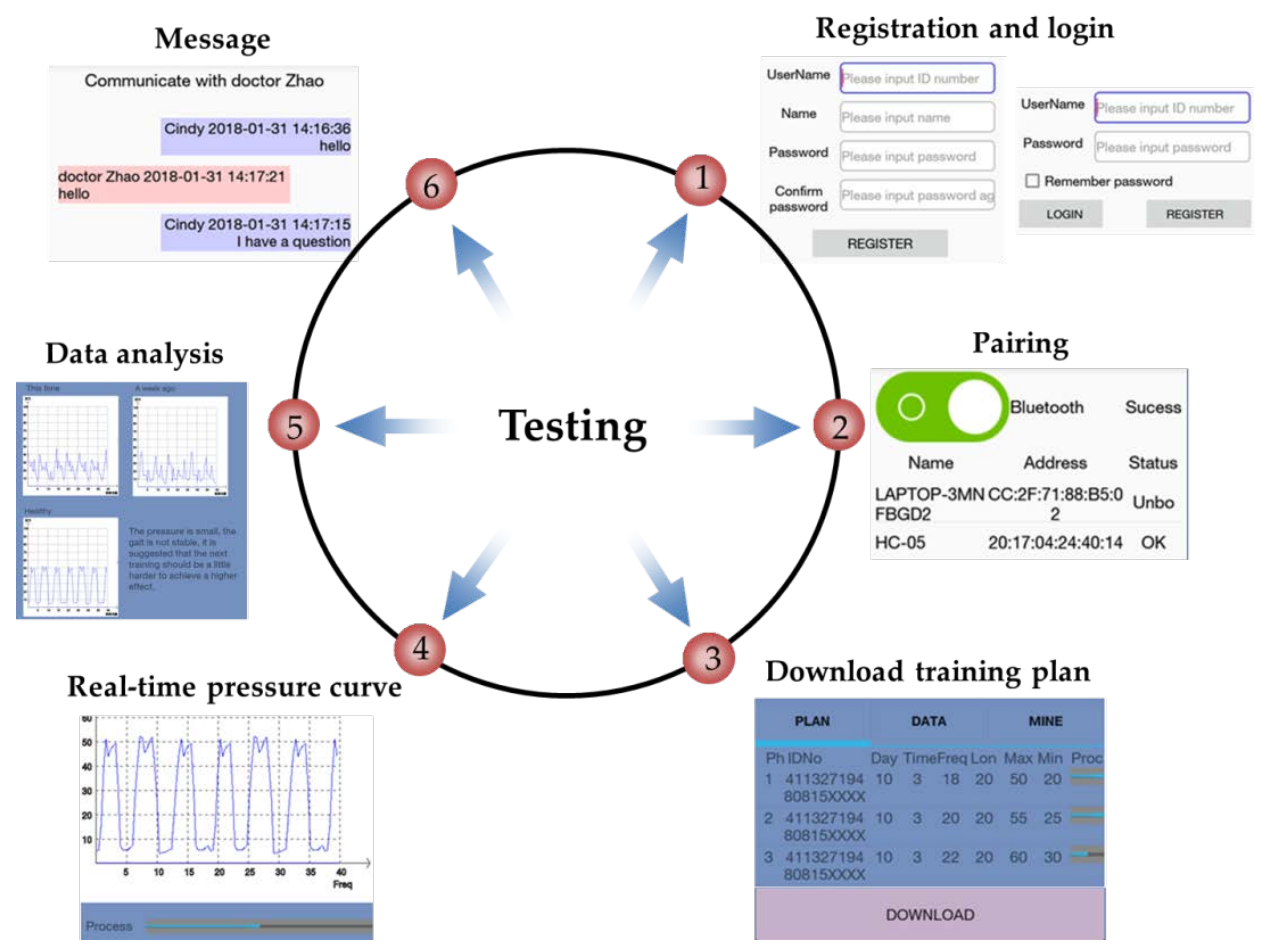

Figure 7. Illustrative images of testing process.

\section{Pilot Experiment and Results}

\subsection{Gait Analysis for Healthy Persons}

Gait is an important indicator for studying human health. The commonly used gait conversion process is mainly divided into seven stages [21], as shown in Figure 8.

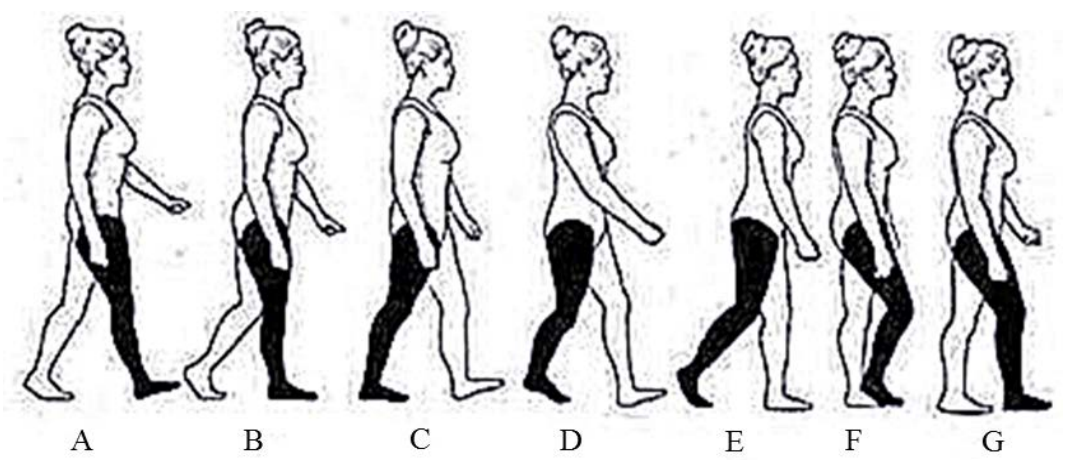

Figure 8. The seven stages of gait conversion process: (A) Foot strike, (B) opposite toe-off, (C) reversal of fore-aft shear, (D) opposite foot strike, (E) toe-off, (F) foot clearance, (G) tibia vertical.

As to a single foot, the gait can be divided into five stages according to Figure 8 (shown in Figure 9):

(A) The rear foot touches the ground;

(B) The entire foot touches the ground;

(C) The rear foot leaves the ground and only forefoot touches the ground;

(D) The entire foot leaves the ground;

(E) The foot strike. 


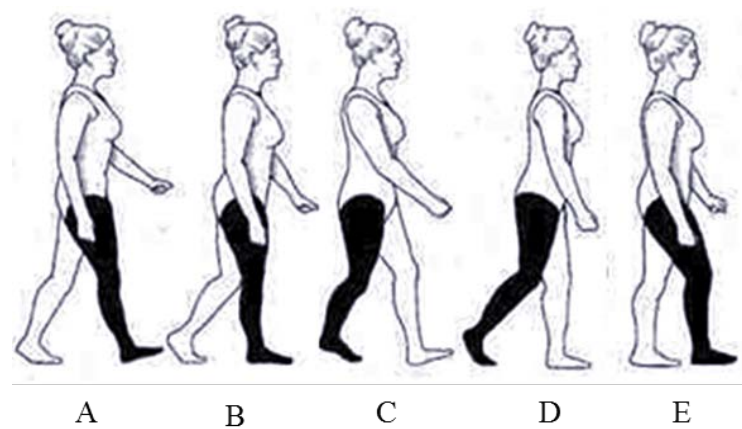

Figure 9. Diagram of gait conversion process. (A) Rear foot touch; (B) Entire foot touch; (C) Rear foot leave and forefoot touch; (D) Entire foot leave; (E) Foot strike.

Ten healthy persons were invited to participate in this testing, each of them exercised 10 times for $2 \mathrm{~min}$ per time, then the typical gaits of the entire foot pressure, forefoot pressure, and rear foot pressure were acquired to analyze the walking characteristics, as shown in Figure 10. The red solid line represents the pressure of whole foot, the blue dot dash line represent $\mathrm{s}$ the fore foot pressure, and the green dashed line represents the rear foot pressure. The five stages (T1-T5) of a single gait can be obtained by analysis the pressures of the whole foot, fore foot and rear foot easily. We can find that the gait conversion process of T1-T5 was consistent with the standard gait conversion process (A)-(E) described above. It shows that the app is able to obtain the plantar pressure of the normal gait for effectively expressing the gait conversion process.

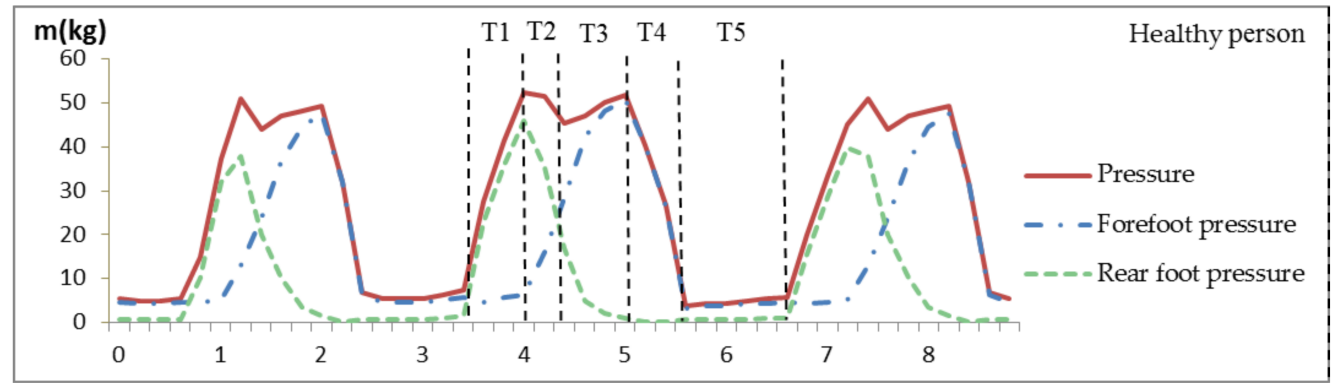

Figure 10. Pressure curves of healthy person gait.

\subsection{Data Acquisition and Gait Analysis of Pilot Experiment}

Patient data acquisition of three lower limb postoperative patients was done in the Beijing Aerospace General Hospital, as shown in Figure 11. The basic information of the three patients is shown in Table 2.
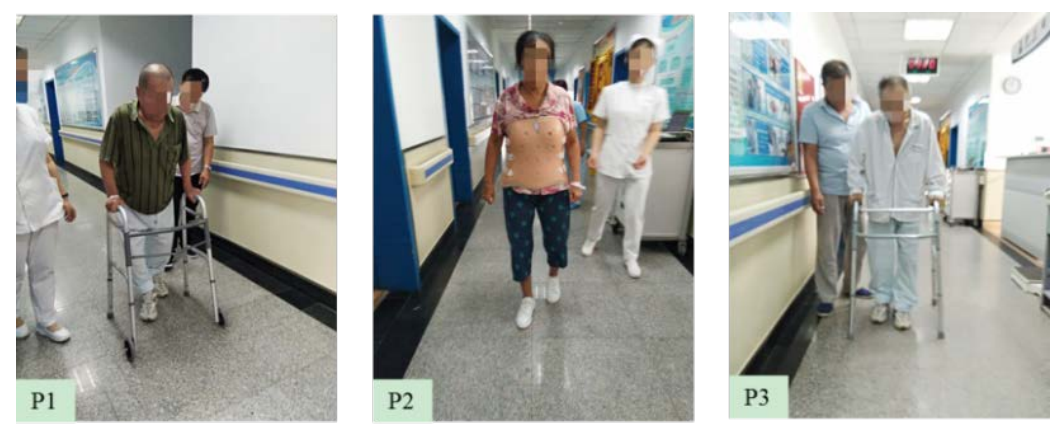

Figure 11. Photographs of the clinical trial. 
Table 2. Characteristics of subject

\begin{tabular}{ccccc}
\hline Subject & Gender & Age & Disease & Rehabilitation Stage \\
\hline P1 & Male & 81 & Femoral neck fracture & 3 months after surgery \\
P2 & Female & 69 & Lumbar spine disease & 2 weeks after surgery \\
P3 & Male & 84 & Femoral neck fracture & 3 weeks after surgery \\
\hline
\end{tabular}

In Figure 12, the typical gait of healthy person is used as the comparison with the gait of the patients. It is easy to find that the gait of the three patients is significantly different from that of the normal persons. The gait of the same disease in different rehabilitation stages is also different to each other. Preliminary testing and pilot experiment show that the app has achieved basic functions, which can obtain accurate and reliable plantar pressure for quantitative gait analysis in the future.

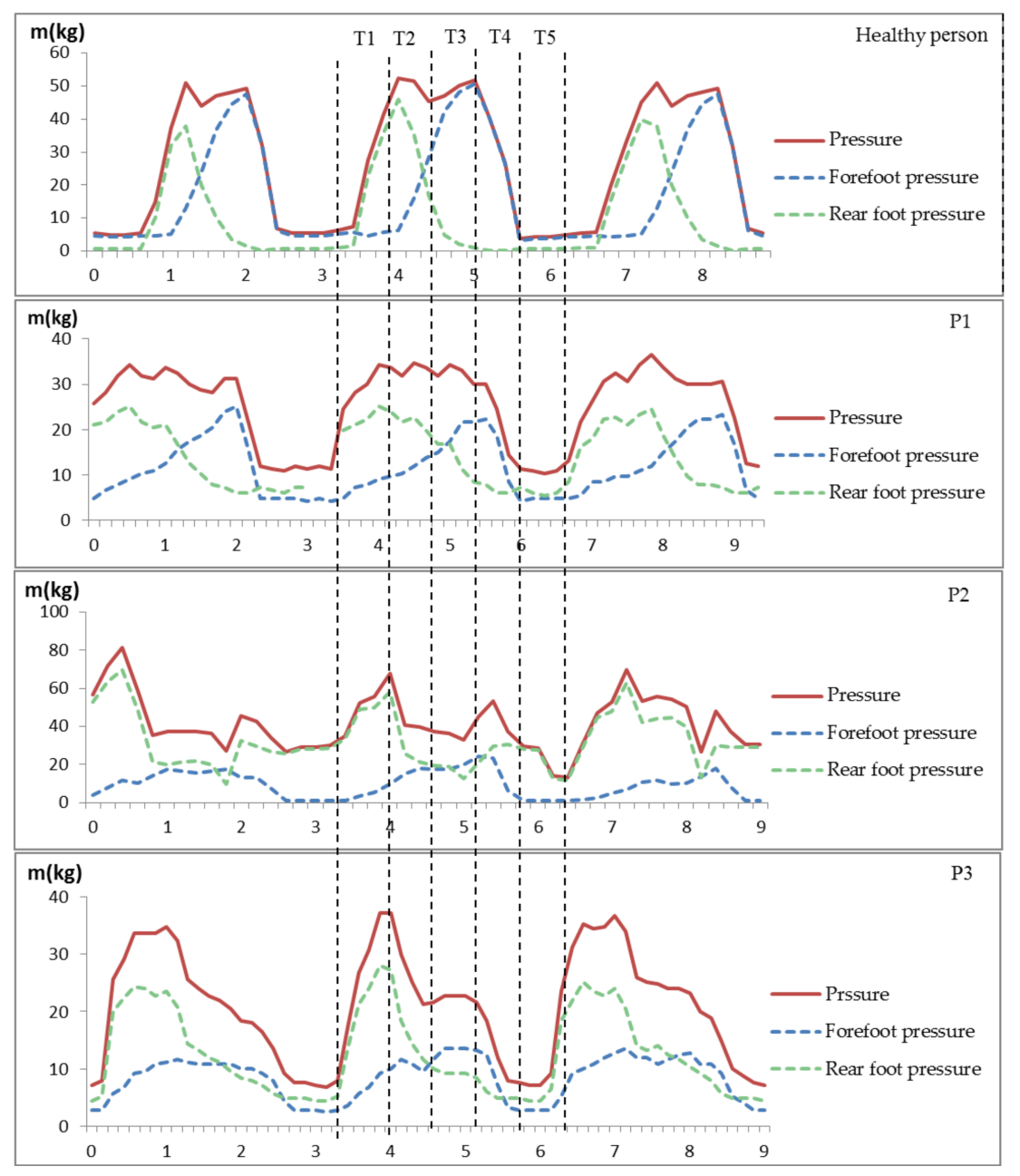

Figure 12. The comparison of normal gait and patients' gaits.

\section{Conclusions and Prospects}

Studies have shown that the earlier rehabilitation training is, the better postoperative results will be. To make patients recover sooner from the hospital, a gait rehabilitation robot assisted training with some quantitatively detected sensor data is the most commonly used training method at present [22,23], but it is mainly applicable to recover in the hospital without a precise and objective evaluation method of training effect $[24,25]$. Therefore, combined with the 'medical + internet' model, an app based on plantar pressure analysis is introduced, which can obtain the doctor's training plan via the internet 
and transmit it to the plantar pressure testing device. The training data can also be obtained from plantar pressure testing device and transferred to the doctor. In addition, patients and doctors can communicate in real time. Preliminary testing and pilot experiment showed that the app can obtain accurate and reliable plantar pressure for quantitative gait analysis. The characteristics of different stages in the same disease can be distinguished clearly. Thus, the rehabilitation laws can be summarized with the support of big data, providing decision basis for doctors to make follow-up training plans.

At the same time, the design of the app provides a new way for patients to recover out of hospitals. For patients, some of the rehabilitation processes in the hospital can be transferred to the family with the help of the app, thus shortening hospital stays and saving costs. They can also learn about their recovery conditions in real time through the app, such as the training phase and training effect. For doctors, they can intuitively see the recovery of patients who are not in the hospital, and direct patient training in real time. In addition, the medical resources can be saved by conducting rehabilitation training for patients at home, making up for the current shortage of medical resources and the imbalance of distribution.

On the other hand, telemedicine and apps are only technical means. To explore the general laws of postoperative rehabilitation by the app we developed is the most important, which is to provide guidance for various types of training and provide a basis for evaluating various rehabilitation techniques. In the future, a lot of experiments shall be done with our designed device and app to obtain more patients' pressure data of traditional recovery in different rehabilitation stages. The laws of postoperative rehabilitation will be worked out and be used as the evaluation method of various rehabilitation techniques with the help of big data and machine learning methods.

Author Contributions: X.C., X.M. and S.K. conceived and designed the experiments. Y.H. performed the experiments. Y.F. and S.W. analyzed the data. F.Y. contributed analysis tools and checked the paper. X.C. wrote the paper.

Acknowledgments: This work has been supported by National Nature Science Foundation of China (no. U1713218, 61375090, U1613224), and National High-Tech R\&D Program (863 Program) (no. 2015AA043204).

Conflicts of Interest: The authors declare no conflict of interest.

\section{References}

1. Yeh, S.C.; Chang, S.M.; Chen, S.Y.; Hwang, W.Y.; Huang, T.C.; Tsai, T.L. A lower limb fracture postoperative-guided interactive rehabilitation training system and its effectiveness analysis. In Proceedings of the 2012 IEEE 14th International Conference on E-Health Networking, Applications and Services, Beijing, China, 10-13 October 2012; pp. 149-154.

2. Lu, Y.; Orthopedics, D.O. The application research of individualized recovery training on postoperative rehabilitation of patients with lower limb fracture. J. Clin. Med. Pract. 2014, 18, 54-56.

3. Du-Liang, X.U.; Huang, J. Influence Factors and Preventive Measures of Lower Extremity Venous Thrombosis after Joint Replacement. Med. Recapitul. 2014, 20, 2596-2598.

4. Titianova, E.B.; Mateev, P.S.; Tarkka, I.M. Footprint analysis of gait using a pressure sensor system. J. Electromyogr. Kinesiol. 2004, 14, 275-281. [CrossRef]

5. Jahss, M.H. Biomechanics of the diabetic foot: A quantitative approach to the assessment of neuropathy, deformity and plantar pressure. Disord. Foot Ankle 1991, 2, 1864-1907.

6. Zhang, X.C.; Zhang, L.X.; Zhao, L.Y. Method for measuring foot applied force in a lower limb rehabilitation training robot. J. Harbin Eng. Univ. 2007, 28, 447-451.

7. Jian-She, L.I.; Wang, L.P. Application and Advances of Sole Pressure Measurement in Biomechanical Research. J. Beijing Univ. Phys. Educ. 2005, 28, 97-124.

8. Ding Min, L.I.; Jian-min, W.U.; Shen, H.T. Research advances and clinical application of lower limb gait rehabilitation robots. J. Clin. Rehabil. Tissue Eng. Res. 2010, 14, 6604.

9. Krebs, H.I.; Volpe, B.T.; Aisen, M.L.; Hogan, N. Increasing productivity and quality of care: Robot-aided neuro-rehabilitation. J. Rehabil. Res. Dev. 2000, 37, 639-652. [PubMed]

10. Colombo, G.; Joerg, M.; Schreier, R.; Dietz, V. Treadmill training of paraplegic patients using a robotic orthosis. J. Rehabil. Res. Dev. 2000, 37, 693-700. [PubMed] 
11. Goffer, A. Gait-Locomotor Apparatus. E.P. Patent 1260201, 10 December 2008.

12. Kwakkel, G.; Kollen, B.J.; Krebs, H.I. Effects of robot-assisted therapy on upper limb recovery after stroke: A systematic review. Neurorehabilit. Neural Repair 2008, 22, 111-121. [CrossRef] [PubMed]

13. Colombo, R.; Pisano, F.; Micera, S.; Mazzone, A.; Delconte, C.; Carrozza, M.C.; Dario, P.; Minuco, G. Assessing mechanisms of recovery during robot-aided neurorehabilitation of the upper limb. Neurorehabil. Neural Repair 2008, 22, 50-63. [CrossRef] [PubMed]

14. Kayama, K.; Yairi, I.E.; Igi, S. Semi-autonomous outdoor mobility support system for elderly and disabled people. In Proceedings of the 2003 IEEE International Conference on Intelligent Robots and Systems, Las Vegas, NV, USA, 27-31 October 2003; Volume 3, pp. 2606-2611.

15. Ye, J.; Huang, J.; He, J.; Tao, C.; Wang, X. Development of a width-changeable intelligent walking-aid robot. In Proceedings of the 2012 IEEE International Symposium on Micro-Nanomechatronics and Human Science, Nagoya, Japan, 4-7 November 2012; pp. 358-363.

16. Ficke, R.C. Digest of Data on Persons with Disabilities; National Institute on Disability \& Rehabilitation Research: Washington, DC, USA, 1992; p. 207.

17. Rajanna, V.; Vo, P.; Barth, J.; Mjelde, M.; Grey, T.; Oduola, C.; Hammond, T. KinoHaptics: An automated, wearable, Haptic assisted, physio-therapeutic system for post-surgery rehabilitation and self-care. J. Med. Syst. 2016, 40, 1-12. [CrossRef] [PubMed]

18. Li, K.; Hao, Y.; Hu, X.; Xie, D.; Li, X.; Zheng, H.; Fu, Y.; Zheng, Y. The effect of sensorimotor training performed by carers on home-based rehabilitation in stroke patients. Physiotherapy 2015, 101, e866-e867. [CrossRef]

19. Ali, I.M.; Samsudin, N. The Design and Development of BMI Calc Android Application. In IOP Conference Series: Materials Science and Engineering; IOP Publishing: Bristol, UK, 2016; Volume 160, p. 012115.

20. Jun, R. Design of Multi-tiered Distributed Software Architecture Based on Patterns. Comput. Eng. 2006, 32, 57-59.

21. Pappas, I.P.; Popovic, M.R.; Keller, T.; Dietz, V.; Morari, M. A reliable gait phase detection system. IEEE Trans. Neural Syst. Rehabil. Eng. 2001, 9, 113-125. [CrossRef] [PubMed]

22. Colombo, G.; Wirz, M.; Dietz, V. Driven gait orthosis for improvement of locomotor training in paraplegic patients. Spinal Cord 2001, 39, 252-255. [CrossRef] [PubMed]

23. Zhang, J.F.; Dong, Y.M.; Yang, C.J.; Geng, Y.; Chen, Y.; Yang, Y. 5-Link model based gait trajectory adaption control strategies of the gait rehabilitation exoskeleton for post-stroke patients. Mechatronics 2010, 20, 368-376. [CrossRef]

24. Peri, E.; Turconi, A.C.; Biffi, E.; Maghini, C.; Panzeri, D.; Morganti, R.; Pedrocchi, A.; Gagliardi, C. Effects of dose and duration of robot-assisted gait training on walking ability of children affected by cerebral palsy. Technol. Health Care 2017, 25, 671-681. [CrossRef] [PubMed]

25. Kim, K.H.; Lee, K.B.; Bae, Y.H.; Fong, S.S.; Lee, S.M. Effects of progressive backward body weight suppoted treadmill training on gait ability in chronic stroke patients: A randomized controlled trial. Technol. Health Care 2017, 25, 867-876. [CrossRef] [PubMed]

(C) 2018 by the authors. Licensee MDPI, Basel, Switzerland. This article is an open access article distributed under the terms and conditions of the Creative Commons Attribution (CC BY) license (http:// creativecommons.org/licenses/by/4.0/). 